\title{
Partial mitochondrial DNA sequences suggest the existence of a cryptic species within the Leucosphyrus group of the genus Anopheles (Diptera: Culicidae), forest malaria vectors, in northern Vietnam
}

\author{
Kohei Takenaka Takano*1,3, Ngoc Thi Hong Nguyen², Binh Thi Huong Nguyen², Toshihiko Sunahara1,
}

Michio Yasunami ${ }^{1}$, Manh Duc Nguyen² and Masahiro Takagi1

\begin{abstract}
Background: During the last decade, Southeast Asian countries have been very successful in reducing the burden of malaria. However, malaria remains endemic in these countries, especially in remote and forested areas. The Leucosphyrus group of the genus Anopheles harbors the most important malaria vectors in forested areas of Southeast Asia. In Vietnam, previous molecular studies have resulted in the identification of only Anopheles dirus sensu stricto (previously known as An. dirus species A) among the Leucosphyrus group members. However, Vietnamese entomologists have recognized that mosquitoes belonging to the Leucosphyrus group in northern Vietnam exhibit morphological characteristics similar to those of Anopheles takasagoensis, which has been reported only from Taiwan. Here, we aimed to confirm the genetic and morphological identities of the members of the Leucosphyrus group in Vietnam.

Results: In the molecular phylogenetic trees reconstructed using partial COI and ND6 mitochondrial gene sequences, samples collected from southern and central Vietnam clustered together with GenBank sequences of An. dirus that were obtained from Thailand. However, samples from northern Vietnam formed a distinct clade separated from both An. dirus and An. takasagoensis by other valid species.

Conclusions: The results suggest the existence of a cryptic species in northern Vietnam that is morphologically similar to, but phylogenetically distant from both An. dirus and An. takasagoensis. We have tentatively designated this possible cryptic species as Anopheles aff. takasagoensis for convenience, until a valid name is assigned. However, it is difficult to distinguish the species solely on the basis of morphological characteristics. Further studies on such as karyotypes and polytene chromosome banding patterns are necessary to confirm whether An. aff. takasagoensis is a valid species. Moreover, studies on (1) the geographic distribution, which is potentially spreading along the Vietnam, China, Laos, and Myanmar borders; (2) morphological and ecological characteristics; and (3) vectorial capacity of this newly identified cryptic species of An. dirus, which is one of the most important malaria vectors in the mainland of Southeast Asia, are necessary for planning efficient malaria vector control programs in this region.
\end{abstract}

\section{Background}

During the last decade, mainland Southeast Asian countries (i.e., Cambodia, Laos, Myanmar, Thailand, and Viet-

\footnotetext{
*Correspondence: koheit@nagasaki-u.ac.jp

1 Institute of Tropical Medicine (NEKKEN), Nagasaki University, Sakamoto,

Nagasaki 852-8523, Japan
}

Full list of author information is available at the end of the article

@ 2010 Takano et al; licensee BioMed Central Ltd. This is an Open Access article distributed under the terms of the Creative Commons BHW 1 Central Attribution License (http://creativecommons.org/licenses/by/2.0), which permits unrestricted use, distribution, and reproduction in any medium, provided the original work is properly cited.

nam) have been very successful in reducing the burden of malaria [1]. Their main strategies included prompt diagnosis and treatment and widespread coverage of vector control through insecticide-treated nets and indoor residual spraying [2]. Malaria, however, has not yet completely disappeared and remains endemic in these coun- 
tries. In remote and forested areas, the transmission rates are still high because of complex interactions between vectors, humans, and environmental factors [3], [4]. Indoor residual spraying is ineffective against vectors that rest outdoors after feeding and vectors encountered outdoors [5], and bed nets are not easily adaptable to the lifestyle of forest workers [4]. Environmental modifications that affect the distribution and abundance of vectors lead to changes in malaria transmission [6]. Under these conditions, accurate species identification is essential in vector control.

The Leucosphyrus group consists of 20 formally described species and 2 informal forms, and its members are distributed in the Oriental region [7-9]. The Dirus complex of the Leucosphyrus group includes the most important malaria vectors in forested areas in mainland Southeast Asia [9], [10]. Anopheles dirus Peyton and Harrison, 1979 was first separated from Anopheles balabacensis Baisas, 1936 [11]. Shortly thereafter, Anopheles takasagoensis Morishita, 1946 was elevated to species status from a synonym of An. balabacensis on the basis of cross-mating, cytogenetic, and morphological evidence [12]. These findings implied that An. balabacensis, which until that time was considered to be the primary vector of human malaria in an area stretching from east India to the Philippines, is not a single species but a complex of three or more species [9]. Mainly on the basis of crossmating and cytogenetic experiments, subsequent intensive studies [8], [13-18] revealed that An. dirus also exists as a species complex that includes at least seven species: $A n$. dirus sensu stricto (previously known as An. dirus species A); Anopheles cracens Sallum and Peyton, 2005 (species B); Anopheles scanloni Sallum and Peyton, 2005 (species C); Anopheles baimaii Sallum and Peyton, 2005 (species D); Anopheles elegans (James), 1903 (species E); Anopheles nemophilous Peyton and Ramalingam, 1988 (species F); and An. takasagoensis. At least two species in the Dirus complex, namely, An. dirus and An. baimaii, are recognized as major malaria vectors [9], [10]. Anopheles balabacensis is now classified into the Leucosphyrus complex [19] with Anopheles leucosphyrus Dönitz, 1901, Anopheles latens Sallum and Peyton, 2005, and Anopheles introlatus Colless, 1957 [7-9] (see also Table 1).

Among the members of the Leucosphyrus group, only An. dirus sensu strict has been found in Vietnam in previous molecular studies (reviewed in [20]). However, a member of the Leucosphyrus group in northern Vietnam had been identified as An. takasagoensis by some Vietnamese entomologists for the last 30 years [21-23]. Here, we conducted a molecular study on members of the Leucosphyrus group in Vietnam to confirm their genetic identity.

Manguin et al. [24] analyzed several specimens collected in 1970 from Ninh Bình Province, northern Viet- nam, which exhibited adult and larval characteristics of both An. dirus and An. takasagoensis. They succeeded in sequencing mitochondrial Cytochrome $c$ oxidase subunit I (COI) and ribosomal DNA internal transcribed spacer 2 (ITS2) of one of the specimens and identified it as $A n$. dirus, whereas they have not yet deposited the sequence into the DDBJ/EMBL/GenBank database. They noted, "This casts doubt on the reported occurrence of $A n$. takasagoensis...in northern Vietnam, but additional material needs to be collected and analysed before it will be known for certain whether the distribution of this species is limited to Taiwan." Here, we conducted a molecular study on members of the Leucosphyrus group in Vietnam to confirm their genetic identity.

Sallum et al. [25] conducted a molecular phylogenetic study of the Leucosphyrus group. For comparison, we chose the same molecular markers: the partial sequences of COI (221 base pair (bp)) and NADH dehydrogenase subunit 6 (ND6: $349 \mathrm{bp}$ ) among mitochondrial genes. The advantage of using the same molecular markers is that Sallum et al. [25] assessed 13 of 20 species of the Leucosphyrus group, including all the members of the Dirus complex. The disadvantages are that these markers cannot distinguish between $A n$. dirus and An. baimaii and that the phylogenetic relationship within the group remains ambiguous, presumably because of the short length of the sequences analyzed (570 bp in total). However, to date, this is the only available molecular information covering the group but is still informative for distinguishing species (except An. dirus and An. baimaii).

\section{Methods}

\section{Mosquito collection and preliminary identification}

In 2008, we conducted field sampling in Bắc Kạn Province for one week and collected 11 larvae (but no adults) of the Leucosphyrus group, eight of which were analyzed in this study (Tables 2 and 3). These larvae were collected from partially or heavily shaded small pools near the starting points (seepage) of small streams in hilly areas covered with secondly evergreen forests. The water of the larval habitats was clear and not running. Other larval and female-adult samples were collected from various parts of Vietnam (Table 1). Collected larvae were reared to obtain adult specimens. Some samples were provided by collaborative entomologists, and a Hai Nan Island (China) strain of $A n$. dirus maintained at the National Institute of Malariology, Parasitology and Entomology was also analyzed. The adult samples were tentatively identified as An. takasagoensis if they had more than one of the following three morphological characteristics (we followed the terminology reported in [26], [27] as well as in [9]): a presector dark spot on vein $\mathrm{R}$ not or barely extending basally beyond the presector pale spot on the costa (basal extension typically occurs in $A n$. dirus), a 
Table 1: In-group and out-group data available from the International Nucleotide Sequence Database with internal classification of the Leucosphyrus group.

\begin{tabular}{|c|c|c|c|c|c|}
\hline \multirow[b]{2}{*}{$\begin{array}{l}\text { Specific name_ID in } \\
\text { Sallum et al. [22] }\end{array}$} & \multicolumn{2}{|c|}{ Accession numbers } & \multirow[b]{2}{*}{ Haplo-type } & \multirow[b]{2}{*}{ Complex } & \multirow[b]{2}{*}{ Subgroup } \\
\hline & $\mathrm{COI}$ & ND6 & & & \\
\hline latens_2 & DQ897936 & DQ899796 & 23 & Leucosphyrus & \\
\hline latens_4 & DQ897937 & DQ899797 & 24 & & \\
\hline leucosphyrus_1 & DQ897938 & DQ899798 & 25 & & \\
\hline leucosphyrus_2 & DQ897939 & DQ899799 & 25 & & \\
\hline balabacensis_1 & DQ897940 & DQ899800 & 26 & & \\
\hline balabacensis_2 & DQ897941 & DQ899801 & 27 & & \\
\hline balabacensis_3 & DQ897942 & DQ899802 & 28 & & \\
\hline dirus_3 & DQ897943 & DQ899803 & 29 & Dirus & Leucosphyrus \\
\hline dirus_4 & DQ897944 & DQ899804 & 7 & & \\
\hline dirus_5 & DQ897945 & DQ899805 & 7 & & \\
\hline dirus_6 & DQ897946 & DQ899806 & 7 & & \\
\hline cracens_1 & DQ897947 & DQ899807 & 30 & & \\
\hline cracens_2 & DQ897948 & DQ899808 & 31 & & \\
\hline scanloni_2 & DQ897949 & DQ899809 & 32 & & \\
\hline scanloni_4 & DQ897950 & DQ899810 & 33 & & \\
\hline scanloni_5 & DQ897951 & DQ899811 & 34 & & \\
\hline baimaii_2 & DQ897952 & DQ899812 & 6 & & \\
\hline baimaii_3 & DQ897953 & DQ899813 & 7 & & \\
\hline baimaii_4 & DQ897954 & DQ899814 & 7 & & \\
\hline baimaii_5 & DQ897955 & DQ899815 & 35 & & \\
\hline baimaii_6 & DQ897956 & DQ899816 & 36 & & \\
\hline elegans_1 & DQ897957 & DQ899817 & 37 & & \\
\hline elegans_3 & DQ897958 & DQ899818 & 37 & & \\
\hline nemophilous_1 & DQ897959 & DQ899819 & 38 & & \\
\hline nemophilous_3B & DQ897960 & DQ899820 & 39 & & \\
\hline nemophilous_4 & DQ897961 & DQ899821 & 40 & & \\
\hline takasagoensis_1 & DQ897962 & DQ899822 & 41 & & \\
\hline takasagoensis_2 & DQ897963 & DQ899823 & 41 & & \\
\hline takasagoensis_3 & DQ897964 & DQ899824 & 42 & & \\
\hline mirans_1 & DQ897965 & DQ899825 & 43 & & Hackeri \\
\hline mirans_3 & DQ897966 & DQ899826 & 44 & & \\
\hline sulawesi & DQ897967 & DQ899827 & 45 & & \\
\hline macarthuri_1 & DQ897968 & DQ899828 & 46 & & Riparis \\
\hline macarthuri_2 & DQ897969 & DQ899829 & 47 & & \\
\hline macarthuri_3 & DQ897970 & DQ899830 & 48 & & \\
\hline macarthuri_5 & DQ897971 & DQ899831 & 48 & & \\
\hline macarthuri_6 & DQ897972 & DQ899832 & 48 & & \\
\hline
\end{tabular}


Table 1: In-group and out-group data available from the International Nucleotide Sequence Database with internal classification of the Leucosphyrus group. (Continued)

\begin{tabular}{lccc}
\hline gambiae & L20934 & L20934 & 49 \\
quadrimaculatus A & NC_000875 & NC_000875 & 50 \\
albimanus & AF417695 & U35259 & 51 \\
aquasalis & AF417697 & U35260 & 52 \\
\hline
\end{tabular}

Anopheles gambiae, An. quadrimaculatus A, An. albimanus, and An. aquasalis were assigned into an out-group, and the remaining species, as well as samples obtained in the present study, were assigned into an in-group.

pale fringe spot between veins $1 \mathrm{~A}$ and $\mathrm{Cu}_{2}$ present on at least one wing (absent in An. dirus), and an accessory sector pale spot on the subcosta (absent in An. dirus) (Table 1 and Figure 1).

\section{DNA extraction}

The specimens had been stored dry at room temperature or below $-20^{\circ} \mathrm{C}$ for up to 7 years prior to DNA extraction. Depending on the condition of each specimen, we used a single leg or a combination of a single leg and some other body parts (i.e., additional legs, a wing, or a head, but not female abdomens, the spermathecae of which might include sperm from mating). We extracted DNA using the REDExtract-N-Amp ${ }^{\text {Tw }}$ Tissue PCR Kit (SigmaAldrich) with a modification of the manufacturer's protocol for animal tissues. Extraction Solution and Tissue Preparation Solution were mixed in a 4:1 ratio. We added $20 \mu \mathrm{l}$ of the mixture per leg and homogenized them in a microtube using the tip of a pipette. After $10 \mathrm{~min}$ of incubation at room temperature, the samples were incubated at $95^{\circ} \mathrm{C}$ for $3 \mathrm{~min}$. We added $16 \mu \mathrm{l}$ of Neutralization Solution B per $20 \mu \mathrm{l}$ of the homogenized sample and mixed them by vortexing. The neutralized tissue extract was centrifuged at 10,000 $g$ for $1 \mathrm{~min}$, and the supernatant was added to a new microtube and stored at $4^{\circ} \mathrm{C}$ until used for PCR reactions.

\section{Markers and primers used in this study}

We selected molecular markers and primers according to Sallum et al [22]. Amplification with the primers UEA9.2 (5'-cta aca ttt ttt cct caa cat ttt tta gg-3') and UEA10.2 (5'tta tta gtt aat aay ggt art tct g-3') yielded a 221-bp product (excluding the primers), partial sequence of the COI gene. Amplification of the primers ND6.F2 (5'-ttg gwc gta awg gwc cat aaa a-3') and ND6.R3 (5'-car gaa tyt atg taa aaa cat ttt $\left.\mathrm{g}-3^{\prime}\right)$ resulted in a product of $349 \mathrm{bp}$ (excluding the primers), part of the ND6 gene.

\section{$P C R$, sequencing, and alignment}

We modified the PCR protocol of Sallum et al. [25]. For COI, each 20- $\mu \mathrm{l}$ PCR reaction contained $2 \mu \mathrm{l}$ of $10 \times \mathrm{EX}$ Taq buffer (TaKaRa, Japan), $0.2 \mu \mathrm{M}$ of dNTP, $0.5 \mu \mathrm{M}$ of UEA9.2 primer, $1.0 \mu \mathrm{M}$ of UEA10.2 primer, $0.1 \mu \mathrm{l}$ of EX Taq ${ }^{\circ}$ Hot Start Version (TaKaRa), and 1.0 to $2.0 \mu$ of DNA extract. The reaction mixture for ND6 was the same as that for COI, except that $1.0 \mu \mathrm{M}$ each of ND6F.2 and ND6.R3 primer was added instead of UEA9.2 and UEA10.2. ASTEC PC320 and PC816 thermal cyclers were used. The thermal cycling profile for $\mathrm{COI}$ consisted of 5 cycles of $30 \mathrm{~s}$ at $94^{\circ} \mathrm{C}, 30 \mathrm{~s}$ at $37^{\circ} \mathrm{C}$, and $30 \mathrm{~s}$ at $72^{\circ} \mathrm{C}$, followed by 40 cycles of $30 \mathrm{~s}$ at $94^{\circ} \mathrm{C}, 30 \mathrm{~s}$ at $47^{\circ} \mathrm{C}$, and $30 \mathrm{~s}$ at $72^{\circ} \mathrm{C}$, with a final extension of $2 \mathrm{~min}$ at $72^{\circ} \mathrm{C}$. The profile for ND6 consisted of 5 cycles of $30 \mathrm{~s}$ at $94^{\circ} \mathrm{C}, 30 \mathrm{~s}$ at $37^{\circ} \mathrm{C}$, and $30 \mathrm{~s}$ at $72^{\circ} \mathrm{C}$, followed by 45 cycles of $30 \mathrm{~s}$ at $94^{\circ} \mathrm{C}, 30 \mathrm{~s}$ at $49^{\circ} \mathrm{C}$, and $30 \mathrm{~s}$ at $72^{\circ} \mathrm{C}$, with a final extension of $2 \mathrm{~min}$ at $72^{\circ} \mathrm{C}$. The PCR product was separated on a $2 \%$ agarose gel and visualized by ethidium bromide staining. Fragment sizes and product density were estimated by comparison with molecular weight standards. We purified the PCR products using ExoSAP-IT (GE Healthcare Japan). We diluted ExoSAP-IT 10 times with Milli-Q water, added $2 \mu \mathrm{l}$ of the dilution to $5 \mu \mathrm{l}$ of the PCR product, incubated the solution at $37^{\circ} \mathrm{C}$ for $30 \mathrm{~min}$, and then inactivated enzymes by incubating at $80^{\circ} \mathrm{C}$ for $15 \mathrm{~min}$. Sequence reactions were carried out on both strands of DNA using the primers listed above and the ABI BigDye terminator Cycle Sequencing Kit v3.1 (Applied Biosystems). The reaction products were purified by ethanol precipitation and resolved in $\mathrm{Hi}-\mathrm{Di}^{\mathrm{mm}}$ Formamide following the manufacturer's protocol, and the sequences were determined with an ABI PRISM 3730 Genetic Analyzer. Complimentary strands were combined into consensus sequences, and questionable base calls were corrected manually by comparison with the original waveform. When the correction of the questionable base call was difficult, the site was recorded as missing data. We concatenated the COI and ND6 sequences and identified 22 unique sequences, i.e., haplotypes (Tables 2 and 3 ).

Further, we obtained the GenBank sequences of members of the Leucosphyrus group (in-group, 37 samples) and those of four other Anopheles species (out-group, Table 1). Sallum et al. [25] deposited the COI sequences that include the sequence of the UEA9.2 primer (29 bp) in GenBank and reconstructed phylogenetic trees on the basis of these sequences. We excluded the UEA9.2 primer sequence from our analysis. There were no insertions or deletions in these sequences; however, the sequences 


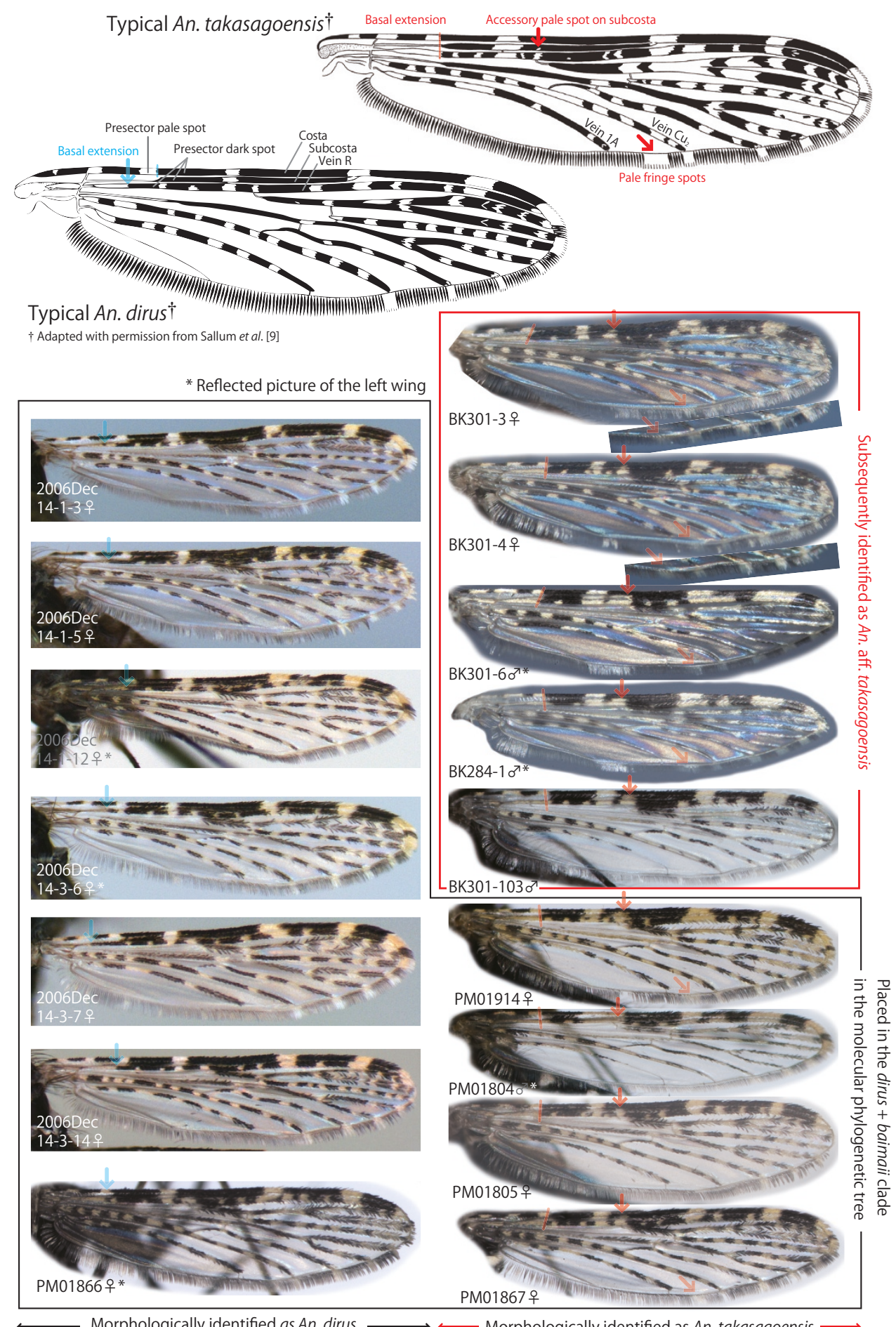

Figure 1 Comparison of wing-spot patterns (dorsal view). Typical An. takasagoensis (upper right), typical An. dirus (upper left, both adapted with permission from Sallum et al. [9]), An. dirus analyzed in this study (black border), and An. aff. takasagoensis (red border). Anopheles aff. takasagoensis exhibited the same spot patterns as typical An. takasagoensis: presector dark spot on vein $\mathrm{R}$ that does not extend or barely extends basally beyond the presector pale spot on the costa, a pale fringe spot between veins $1 \mathrm{~A}$ and $\mathrm{Cu}_{2}$, and an accessory sector pale spot on the subcosta. Some samples from Nghệ An Province (PM01914, PM01804, PM01805, and PM01867) also exhibited spot patterns similar to those of An. takasagoensis, but they were identified as An. dirus by molecular analyses. 
Table 2: Specimens used in this study (to be continued).

\begin{tabular}{|c|c|c|c|c|c|c|c|c|c|}
\hline \multirow[t]{2}{*}{ Specimen ID } & \multirow{2}{*}{$\begin{array}{l}\text { Morphological } \\
\text { identification }\end{array}$} & \multirow[t]{2}{*}{ Sex } & \multirow[t]{2}{*}{ Collection date } & \multirow[t]{2}{*}{ Collector } & \multirow[t]{2}{*}{ Specimen ID } & \multirow[t]{2}{*}{ Latitude } & \multirow[t]{2}{*}{ Longitude } & \multicolumn{2}{|c|}{ Place name in Vietnam } \\
\hline & & & & & & & & Area & Province \\
\hline S1 & An. dirus & M & $24-x-2005$ & Sunahara T. et al. & S1 & $11^{\circ} 59^{\prime} 36.12^{\prime \prime} \mathrm{N}$ & $107^{\circ} 18^{\prime} 13.14^{\prime \prime E}$ & $\begin{array}{l}\text { Southern } \\
\text { Vietnam }\end{array}$ & Bình Phuớc \\
\hline S2 & An. dirus & M & $24-x-2005$ & Sunahara T. et al. & S2 & $11^{\circ} 59^{\prime} 36.12^{\prime \prime} \mathrm{N}$ & $107^{\circ} 18^{\prime} 13.14^{\prime \prime E}$ & & Bình Phuớc \\
\hline S5 & An. dirus & $\mathrm{F}$ & $25-x-2005$ & Sunahara T. et al. & S5 & $11^{\circ} 59^{\prime} 30.84^{\prime \prime} \mathrm{N}$ & $107^{\circ} 18^{\prime} 12.12 " \mathrm{E}$ & & Bình Phuớc \\
\hline S6 & An. dirus & $\mathrm{F}$ & $25-x-2005$ & Sunahara T. et al. & S6 & $11^{\circ} 59^{\prime} 30.84^{\prime \prime} \mathrm{N}$ & $107^{\circ} 18^{\prime} 12.12 " \mathrm{E}$ & & Bình Phuớc \\
\hline S7 & An. dirus & $\mathrm{F}$ & $8-x i-2005$ & Sunahara T. et al. & S7 & $11^{\circ} 05^{\prime} 26.35^{\prime \prime} \mathrm{N}$ & $107^{\circ} 53^{\prime} 59.43 " \mathrm{E}$ & & Bình Thuận \\
\hline S8 & An. dirus & $\mathrm{F}$ & $8-x i-2005$ & Sunahara T. et al. & S8 & $11^{\circ} 05^{\prime} 26.35^{\prime \prime} \mathrm{N}$ & $107^{\circ} 53^{\prime} 59.43 " \mathrm{E}$ & & Bình Thuận \\
\hline S9 & An. dirus & M & $8-x i-2005$ & Sunahara T. et al. & S9 & $11^{\circ} 05^{\prime} 26.35^{\prime \prime} \mathrm{N}$ & $107^{\circ} 53^{\prime} 59.43 " \mathrm{E}$ & & Bình Thuận \\
\hline S11 & An. dirus & $\mathrm{F}$ & $13-x i i-2006$ & Sunahara T. et al. & S11 & $11^{\circ} 42^{\prime} 49.58^{\prime \prime} \mathrm{N}$ & $106^{\circ} 56^{\prime} 02.51 " \mathrm{E}$ & & Bình Phuớc \\
\hline S12 & An. dirus & $\mathrm{F}$ & $19-x i i-2006$ & Sunahara T. et al. & $\mathrm{S} 12$ & $11^{\circ} 42^{\prime} 47.06^{\prime \prime} \mathrm{N}$ & $106^{\circ} 56^{\prime} 54.08^{\prime \prime} \mathrm{E}$ & & Bình Phuớc \\
\hline S13 & An. dirus & $\mathrm{F}$ & $19-x i i-2006$ & Sunahara T. et al. & $\mathrm{S} 13$ & $11^{\circ} 42^{\prime} 47.06^{\prime \prime} \mathrm{N}$ & $106^{\circ} 56^{\prime} 54.08 " \mathrm{E}$ & & Bình Phuớc \\
\hline V24 & An. dirus & $\mathrm{F}$ & 2002 & Nguyen D. M. et al. & V24 & $12^{\circ} 22^{\prime} 21.00^{\prime \prime} \mathrm{N}$ & $109^{\circ} 05^{\prime} 4.12 " \mathrm{E}$ & & Khánh Hòa \\
\hline V25 & An. dirus & $\mathrm{F}$ & 2002 & Nguyen D. M. et al. & V25 & $12^{\circ} 22^{\prime} 21.00^{\prime \prime} \mathrm{N}$ & $109^{\circ} 05^{\prime} 4.12 " \mathrm{E}$ & & Khánh Hòa \\
\hline V27 & An. dirus & $\mathrm{F}$ & 2002 & Nguyen D. M. et al. & V27 & $12^{\circ} 22^{\prime} 21.00^{\prime \prime} \mathrm{N}$ & $109^{\circ} 05^{\prime} 4.12 " \mathrm{E}$ & & Khánh Hòa \\
\hline V43 & An. dirus & $\mathrm{F}$ & 2003 & Nguyen D. M. et al. & V43 & & & & Bình Phuớc \\
\hline V51 & An. dirus & $\mathrm{F}$ & 2007 & Vu Dinh Chu et al. & V51 & $13^{\circ} 08^{\prime} \mathrm{N}$ & $108^{\circ} 50^{\prime} \mathrm{E}$ & & Phú Yên \\
\hline V52 & An. dirus & $\mathrm{F}$ & 2007 & Vu Dinh Chu et al. & V52 & $13^{\circ} 08^{\prime} \mathrm{N}$ & $108^{\circ} 50^{\prime} \mathrm{E}$ & & Phú Yên \\
\hline V53 & An. dirus & $\mathrm{F}$ & 2007 & Vu Dinh Chu et al. & V53 & $13^{\circ} 08^{\prime} \mathrm{N}$ & $108^{\circ} 50^{\prime} \mathrm{E}$ & & Phú Yên \\
\hline V54 & An. dirus & $\mathrm{F}$ & 2007 & Vu Dinh Chu et al. & V54 & $13^{\circ} 08^{\prime} \mathrm{N}$ & $108^{\circ} 50^{\prime} \mathrm{E}$ & & Phú Yên \\
\hline V71 & An. dirus & $\mathrm{F}$ & 2003 & Nguyen D. M. et al. & V71 & $11^{\circ} 05^{\prime} \mathrm{N}$ & $107^{\circ} 54^{\prime} \mathrm{E}$ & & Bình Thuận \\
\hline V72 & An. dirus & $\mathrm{F}$ & 2003 & Nguyen D. M. et al. & V72 & $11^{\circ} 05^{\prime} \mathrm{N}$ & $107^{\circ} 54^{\prime} \mathrm{E}$ & & Bình Thuận \\
\hline V73 & An. dirus & $\mathrm{F}$ & 2003 & Nguyen D. M. et al. & V73 & $11^{\circ} 05^{\prime} \mathrm{N}$ & $107^{\circ} 54^{\prime} \mathrm{E}$ & & Bình Thuận \\
\hline V74 & An. dirus & $\mathrm{F}$ & 2005 & Nguyen Van Chau et al. & V74 & $11^{\circ} 30^{\prime} \mathrm{N}$ & $107^{\circ} 20^{\prime} \mathrm{E}$ & & Đống Nai \\
\hline V76 & An. dirus & $\mathrm{F}$ & 2005 & Nguyen Van Chau et al. & V76 & $11^{\circ} 30^{\prime} \mathrm{N}$ & $107^{\circ} 20^{\prime} \mathrm{E}$ & & Đống Nai \\
\hline 2006Dec14-1-3 & An. dirus & $\mathrm{F}$ & 14-xii-2006 & Takano T. K. et al. & 2006Dec14-1-3 & $11^{\circ} 42^{\prime} 45.9^{\prime \prime} \mathrm{N}$ & $106^{\circ} 56^{\prime} 01.7 " \mathrm{E}$ & & Bình Phuớc \\
\hline 2006Dec14-1-5 & An. dirus & $\mathrm{F}$ & 14-xii-2006 & Takano T. K. et al. & 2006Dec14-1-5 & $11^{\circ} 42^{\prime} 45.9^{\prime \prime} \mathrm{N}$ & $106^{\circ} 56^{\prime} 01.7 " \mathrm{E}$ & & Bình Phuớc \\
\hline 2006Dec14-1-12 & An. dirus & $\mathrm{F}$ & 14-xii-2006 & Takano T. K. et al. & 2006Dec14-1-12 & $11^{\circ} 42^{\prime} 45.9^{\prime \prime} \mathrm{N}$ & $106^{\circ} 56^{\prime} 01.7 " \mathrm{E}$ & & Bình Phuớc \\
\hline 2006Dec14-3-6 & An. dirus & $\mathrm{F}$ & 14-xii-2006 & Takano T. K. et al. & 2006Dec14-3-6 & $11^{\circ} 42^{\prime} 57.9 " \mathrm{~N}$ & $106^{\circ} 56^{\prime} 01.2^{\prime \prime E}$ & & Bình Phuớc \\
\hline
\end{tabular}


Table 2: Specimens used in this study (to be continued). (Continued)

\begin{tabular}{|c|c|c|c|c|c|c|c|c|c|}
\hline 2006Dec14-3-7 & An. dirus & $\mathrm{F}$ & 14-xii-2006 & Takano T. K. et al. & 2006Dec14-3-7 & $11^{\circ} 42^{\prime} 57.9^{\prime \prime} \mathrm{N}$ & $106^{\circ} 56^{\prime} 01.2^{\prime \prime E}$ & & Bình Phuớc \\
\hline 2006Dec14-3-14 & An. dirus & $\mathrm{F}$ & 14-xii-2006 & Takano T. K. et al. & 2006Dec14-3-14 & $11^{\circ} 42^{\prime} 57.9^{\prime \prime} \mathrm{N}$ & $106^{\circ} 56^{\prime} 01.2 " \mathrm{E}$ & & Bình Phuớc \\
\hline Hai Nan strain & An. dirus & & 2008 & & Hai Nan strain & $19^{\circ} 7 ' 25.03^{\prime \prime} \mathrm{N}$ & $109^{\circ} 34^{\prime} 4.05^{\prime \prime E}$ & China & \\
\hline PM01866 & An. dirus & $\mathrm{F}$ & $x-2006$ & Vu Duc Chinh et al. & PM01866 & $19^{\circ} 02^{\prime} \mathrm{N}$ & $104^{\circ} 48^{\prime} \mathrm{E}$ & Central Vietnam & Nghệ An \\
\hline PM01867 & An. takasagoensis & $\mathrm{F}$ & $x-2006$ & Vu Duc Chinh et al. & PM01867 & $19^{\circ} 02^{\prime} \mathrm{N}$ & $104^{\circ} 48^{\prime} \mathrm{E}$ & & Nghệ An \\
\hline PM01804 & An. takasagoensis & M & $x-2006$ & Vu Duc Chinh et al. & PM01804 & $19^{\circ} 02^{\prime} \mathrm{N}$ & $104^{\circ} 48^{\prime} \mathrm{E}$ & & Nghệ An \\
\hline PM01805 & An. takasagoensis & $\mathrm{F}$ & $x-2006$ & Vu Duc Chinh et al. & PM01805 & $19^{\circ} 02^{\prime} \mathrm{N}$ & $104^{\circ} 48^{\prime} \mathrm{E}$ & & Nghệ An \\
\hline PM01914 & An. takasagoensis & $\mathrm{F}$ & $x-2006$ & Vu Duc Chinh et al. & PM01914 & $19^{\circ} 02^{\prime} \mathrm{N}$ & $104^{\circ} 48^{\prime} \mathrm{E}$ & & Nghệ An \\
\hline BK101 & An. takasagoensis & $\mathrm{F}$ & ix-2007 & Le Xuan Hoi et al. & BK101 & $22^{\circ} 05^{\prime} 59.21 " \mathrm{~N}$ & $106^{\circ} 01 ' 26.00^{\prime \prime} \mathrm{E}$ & $\begin{array}{l}\text { Northern } \\
\text { Vietnam }\end{array}$ & Bắc Kạn \\
\hline BK284-2 & An. takasagoensis & M & $8-x-2008$ & Nguyen D. M. & BK284-2 & $22^{\circ} 05^{\prime} 54.29^{\prime \prime} \mathrm{N}$ & $106^{\circ} 01 ' 20.71 " \mathrm{E}$ & & Bắc Kạn \\
\hline BK301-6 & An. takasagoensis & M & $10-x-2008$ & Hoang Van Tan et al. & BK301-6 & $22^{\circ} 05^{\prime} 40.31 " \mathrm{~N}$ & $106^{\circ} 02^{\prime} 17.52^{\prime \prime E}$ & & Bắc Kạn \\
\hline BK301-7 & An. takasagoensis & M & $10-x-2008$ & Hoang Van Tan et al. & BК301-7 & $22^{\circ} 05^{\prime} 40.31 " \mathrm{~N}$ & $106^{\circ} 02^{\prime} 17.52^{\prime \prime E}$ & & Bắc Kạn \\
\hline BK301-103 & An. takasagoensis & M & $14-x-2008$ & Tsuzuki ataru et al. & BK301-103 & $22^{\circ} 05^{\prime} 40.31 " \mathrm{~N}$ & $106^{\circ} 02^{\prime} 17.52^{\prime \prime} \mathrm{E}$ & & Bắc Kạn \\
\hline BK284-1 & An. takasagoensis & M & $8-x-2008$ & Nguyen D. M. & BK284-1 & $22^{\circ} 05^{\prime} 54.29^{\prime \prime} \mathrm{N}$ & 10601'20.71"E & & Bắc Kạn \\
\hline ВК301-3 & An. takasagoensis & $\mathrm{F}$ & $10-x-2008$ & Hoang Van Tan et al. & BK301-3 & $22^{\circ} 05^{\prime} 40.311 " \mathrm{~N}$ & $106^{\circ} 02^{\prime} 17.52^{\prime \prime E}$ & & Bắc Kạn \\
\hline BK301-4 & An. takasagoensis & $\mathrm{F}$ & $10-x-2008$ & Hoang Van Tan et al. & BK301-4 & $22^{\circ} 05^{\prime} 40.31 " \mathrm{~N}$ & $106^{\circ} 02^{\prime} 17.52^{\prime \prime E}$ & & Bắc Kạn \\
\hline
\end{tabular}


Table 3: Specimens used in this study (continued).

\begin{tabular}{|c|c|c|c|c|c|c|c|c|}
\hline \multirow[b]{2}{*}{ Specimen ID } & \multicolumn{3}{|c|}{ Place name in Vietnam } & \multirow[b]{2}{*}{ Remarks } & \multirow[b]{2}{*}{ Haplo-type } & \multicolumn{2}{|c|}{ DDBJ Accession No. } & \multirow[b]{2}{*}{$\begin{array}{c}\text { Molecular } \\
\text { identification }\end{array}$} \\
\hline & District & Commune & Others & & & $\mathrm{COI}$ & ND6 & \\
\hline S1 & Bù Đăng & Đắk Nhau & Đắk Liên & Larval collection & 1 & AB518499 & AB518539 & An. dirus \\
\hline S2 & Bù Đăng & Đắk Nhau & Đắk Liên & Larval collection & 2 & AB518500 & AB518540 & An. dirus \\
\hline S5 & Bù Đăng & Đắk Nhau & Đắk Liên & Larval collection & 3 & AB518501 & AB518541 & An. dirus \\
\hline S6 & Bù Đăng & Đắk Nhau & Đắk Liên & Larval collection & 3 & AB518502 & AB518542 & An. dirus \\
\hline S7 & Hàm Thuận Nam & MY̌ Thanh & & Larval collection & 4 & AB518503 & AB518543 & An. dirus \\
\hline S8 & Hàm Thuận Nam & MY̌ Thanh & & Larval collection & 5 & AB518504 & AB518544 & An. dirus \\
\hline S9 & Hàm Thuận Nam & MY̌ Thanh & & Larval collection & 5 & AB518505 & AB518545 & An. dirus \\
\hline S11 & Phuớc Long & Phú Riêng & Phú Thuận & Indoor light trap & 6 & AB518506 & AB518546 & An. dirus \\
\hline $\mathrm{S} 12$ & Phuớc Long & Phú Riêng & Phú Thuận & Indoor light trap & 7 & AB518507 & AB518547 & An. dirus \\
\hline S13 & Phuớc Long & Phú Riêng & Phú Thuận & Indoor light trap & 6 & AB518508 & AB518548 & An. dirus \\
\hline V24 & Khánh Vinh & Khánh Phú & a forest near Ngã Hai village & $\begin{array}{l}\text { Human landing } \\
\text { catch }\end{array}$ & 7 & AB518509 & AB518549 & An. dirus \\
\hline V25 & Khánh Vinh & Khánh Phú & a forest near Ngã Hai village & $\begin{array}{l}\text { Human landing } \\
\text { catch }\end{array}$ & 7 & AB518510 & AB518550 & An. dirus \\
\hline V27 & Khánh Vinh & Khánh Phú & a forest near Ngã Hai village & $\begin{array}{l}\text { F1 from an adult } \\
\text { female }\end{array}$ & 7 & AB518511 & AB518551 & An. dirus \\
\hline V43 & & & & & 8 & AB518512 & AB518552 & An. dirus \\
\hline V51 & Son Hòa & Ea Chà Rang & Kiến Thiết village & $\begin{array}{l}\text { Human landing } \\
\text { catch }\end{array}$ & 9 & AB518513 & AB518553 & An. dirus \\
\hline V52 & Son Hòa & Ea Chà Rang & Kiến Thiết village & $\begin{array}{l}\text { Human landing } \\
\text { catch }\end{array}$ & 9 & AB518514 & AB518554 & An. dirus \\
\hline V53 & Son Hòa & Ea Chà Rang & Kiến Thiết village & $\begin{array}{l}\text { Human landing } \\
\text { catch }\end{array}$ & 9 & AB518515 & AB518555 & An. dirus \\
\hline V54 & Son Hòa & Ea Chà Rang & Kiến Thiết village & $\begin{array}{l}\text { Human landing } \\
\text { catch }\end{array}$ & 9 & AB518516 & AB518556 & An. dirus \\
\hline V71 & Hàm Thuận Nam & MY̌ Thanh & & $\begin{array}{l}\text { Human landing } \\
\text { catch }\end{array}$ & 10 & AB518517 & AB518557 & An. dirus \\
\hline V72 & Hàm Thuận Nam & MY̌ Thanh & & $\begin{array}{l}\text { Human landing } \\
\text { catch }\end{array}$ & 10 & AB518518 & AB518558 & An. dirus \\
\hline V73 & Hàm Thuận Nam & MY̌ Thanh & & $\begin{array}{l}\text { Human landing } \\
\text { catch }\end{array}$ & 7 & AB518519 & AB518559 & An. dirus \\
\hline V74 & Tận Phú & Đắc Lua & Cát Tiên National Park & $\begin{array}{l}\text { Human landing } \\
\text { catch }\end{array}$ & 5 & AB518520 & AB518560 & An. dirus \\
\hline V76 & Tận Phú & Đắc Lua & Cát Tiên National Park & $\begin{array}{l}\text { Human landing } \\
\text { catch }\end{array}$ & 11 & AB518521 & AB518561 & An. dirus \\
\hline
\end{tabular}


Table 3: Specimens used in this study (continued). (Continued)

\begin{tabular}{|c|c|c|c|c|c|c|c|c|}
\hline 2006Dec14-1-3 & Phuớc Long & Phú Riêng & Phú Thuận & Larval collection & 12 & AB518522 & AB518562 & An. dirus \\
\hline 2006Dec14-1-5 & Phuớc Long & Phú Riêng & Phú Thuận & Larval collection & 10 & AB518523 & AB518563 & An. dirus \\
\hline 2006Dec14-1-12 & Phuớc Long & Phú Riêng & Phú Thuận & Larval collection & 7 & AB518524 & AB518564 & An. dirus \\
\hline 2006Dec14-3-6 & Phuớc Long & Phú Riêng & Phú Thuận & Larval collection & 7 & AB518525 & AB518565 & An. dirus \\
\hline 2006Dec14-3-7 & Phuớc Long & Phú Riêng & Phú Thuận & Larval collection & 13 & AB518526 & AB518566 & An. dirus \\
\hline 2006Dec14-3-14 & Phuớc Long & Phú Riêng & Phú Thuận & Larval collection & 7 & AB518527 & AB518567 & An. dirus \\
\hline Hai Nan strain & & $\begin{array}{l}\text { Reared strain in } \\
\text { NIMPE, originated } \\
\text { from Hai Nan } \\
\text { Island, China }\end{array}$ & & & 9 & AB518528 & AB518568 & An. dirus \\
\hline PM01866 & Con Cuông & Chi Khê & Pù Mát National Forest & Larval collection & 14 & AB518529 & AB518569 & An. dirus \\
\hline PM01867 & Con Cuông & Chi Khê & Pù Mát National Forest & Larval collection & 15 & AB518530 & AB518570 & An. dirus \\
\hline PM01804 & Con Cuông & Chi Khê & Pù Mát National Forest & Larval collection & 16 & AB518531 & AB518571 & An. dirus \\
\hline PM01805 & Con Cuông & Chi Khê & Pù Mát National Forest & Larval collection & 16 & AB518532 & AB518572 & An. dirus \\
\hline PM01914 & Con Cuông & Chi Khê & Pù Mát National Forest & Larval collection & 17 & AB518533 & AB518573 & An. dirus \\
\hline BK101 & Na Rì & Quang Phong & Na Ca village & $\begin{array}{l}\text { Collected at } \\
\text { buffalo hat }\end{array}$ & 18 & AB518534 & AB518574 & $\begin{array}{c}\text { An. aff. } \\
\text { takasagoensis }\end{array}$ \\
\hline BK284-2 & Na Rì & Quang Phong & $\mathrm{Na}$ Ca village & Larval collection & 19 & AB518535 & AB518575 & $\begin{array}{c}\text { An. aff. } \\
\text { takasagoensis }\end{array}$ \\
\hline BK301-6 & Na Rì & Quang Phong & $\mathrm{Na}$ Ca village & Larval collection & 20 & AB518536 & AB518576 & $\begin{array}{c}\text { An. aff. } \\
\text { takasagoensis }\end{array}$ \\
\hline BK301-7 & Na Rì & Quang Phong & $\mathrm{Na}$ Ca village & Larval collection & 21 & AB518537 & AB518577 & $\begin{array}{c}\text { An. aff. } \\
\text { takasagoensis }\end{array}$ \\
\hline BK301-103 & Na Rì & Quang Phong & $\mathrm{Na}$ Ca village & Larval collection & 22 & AB518538 & AB518578 & $\begin{array}{c}\text { An. aff. } \\
\text { takasagoensis }\end{array}$ \\
\hline BK284-1 & Na Rì & Quang Phong & $\mathrm{Na}$ Ca village & Larval collection & \multicolumn{4}{|c|}{ Not analyzed but wing spots are shown in Figure 2 . } \\
\hline BK301-3 & Na Rì & Quang Phong & $\mathrm{Na}$ Ca village & Larval collection & \multicolumn{4}{|c|}{ Not analyzed but wing spots are shown in Figure 2 . } \\
\hline BK301-4 & Na Rì & Quang Phong & $\mathrm{Na}$ Ca village & Larval collection & \multicolumn{4}{|c|}{ Not analyzed but wing spots are shown in Figure 2 . } \\
\hline
\end{tabular}


BK101, BK284-2, BK301-103 (Table 2), balabacensis_3, dirus_3, and baimaii_6 (Table 1) had 8-87 missing sites at the 3'- or 5'-end of either the COI or ND6 sequences (Additional files 1, 2 and 3). Finally, we obtained 52 unique sequences and assigned a haplotype to each of them (Tables 1, 2 and 3 and Additional files 1, 2 and 3).

\section{Molecular phylogeny}

The neighbor-joining (NJ) and maximum parsimony (MP) methods were performed with the MEGA4 software [28]. All codon positions were included, and all ambiguous sites were treated as missing data. The resultant trees were rooted using the out-group. In the NJ phylogenetic reconstruction, the evolutionary distances were computed using the Jukes-Cantor method. All sites containing missing data were eliminated only in pairwise sequence comparisons (pairwise deletion option). To assess the reliability of the $\mathrm{NJ}$ tree, the bootstrap test and the interior branch test were performed with 2,000 replicates. In the MP phylogenetic reconstruction, the most parsimonious trees were obtained using the close-neighbor-interchange algorithm at search level 3, in which the initial trees were obtained by random addition of sequences (10,000 replicates). There were 570 sites in the final dataset, of which 137 were parsimony informative. The consensus tree was generated from the 3517 most parsimonious trees. Branches corresponding to partitions reproduced in less than $50 \%$ of trees were collapsed. Branch lengths were calculated using the average pathway method and are expressed in units of the number of changes over the whole sequence. The percentages of parsimonious trees in which the associated taxa clustered together are shown next to the branches.

\section{Results}

By the morphological examination, all the samples collected from Bắc Kạn Province in northern Vietnam and four of five samples from Nghệ An Province in central Vietnam were tentatively identified as An. takasagoensis (Tables 2 and 3 and Figure 1).

In the molecular phylogenetic reconstruction using the NJ method (Figure 2), haplotypes 18-22 of the Bắc Kạn samples formed a distinct clade with high bootstrap (91\%) and interior branch test (97\%) support; this clade was separated from haplotypes of both An. takasagoensis and $A n$. dirus. This clade then clustered with haplotypes 26-28 of An. balabacensis, but the bootstrap and interior branch test support were lower, with values less than $50 \%$. Other haplotypes obtained in the present study (haplotypes 1-17), including those of the Nghệ An samples, were clustered together with those of $A n$. dirus from Thailand and An. baimaii from Thailand, Myanmar, and Bangladesh. The (An. dirus + An. baimaii) clade was subsequently clustered with $A n$. elegans from India with moderate bootstrap support (75\%) and high interior branch test support (95\%) (indicated with an arrow in Figure 2). Subsequently, An. takasagoensis clustered with the $(A n$. dirus $+A n$. baimaii $+A n$. elegans) clade, $A n$. cracens clustered with the $(A n$. dirus $+A n$. baimaii $+A n$. elegans + An. takasagoensis) clade, and An. scanloni clustered with the $(A n$. dirus $+A n$. baimaii $+A n$. elegans + An. takasagoensis $+A n$. cracens) clade with moderate to low bootstrap and interior branch test support (Figure 2). The Dirus complex members, An. balabacensis, and the Bắc Kạn samples formed a clade with high bootstrap (89\%) and interior branch (98\%) support. This clade next combined with the (An. leucosphyrus + An. latens) clade, and the resultant clade corresponded to the Leucosphyrus subgroup. The Leucosphyrus subgroup clade combined with the Hackeri subgroup clade $(A n$. sulawesi + An. mirans), and further combined with the Riparis subgroup clade (An. macarthuri). The NJ topology was consistent with the traditional classification of the Leucosphyrus group [9], except that the Leucosphyrus complex were regarded as paraphyletic taxa (Figure 2).

In the MP tree (Figure 3), haplotypes 18-22 from the Bắc Kạn samples also formed a clade with $100 \%$ consensus; this clade was separated from the haplotypes of both An. takasagoensis and An. dirus. The Bắc Kạn haplotypes then clustered with haplotypes 26-28 of An. balabacensis with $66 \%$ consensus. Other haplotypes obtained in the present study (haplotypes 1-17), including those from the Nghê An samples, were clustered together with those of An. dirus from Thailand and An. baimaii from Thailand, Myanmar, and Bangladesh with $81 \%$ consensus. Subsequently, the topology ((( An. dirus, An. baimaii) An. elegans) An. takasagoensis) A. cracens) was supported by $100 \%$ consensus (indicated by arrows in Figure 3 ). This clade clustered with An. scanloni and the (An. balabacensis + Bắc Kạn samples) clade with $66 \%$ consensus and further combined with the An. nemophilous clade with $100 \%$ consensus. This clade then combined with the ( $A n$. sulawesi $+A n$. mirans: the Hackeri subgroup) $+A n$. mirans: the Riparis subgroup) clade with $100 \%$ consensus, whereas An. leucosphyrus and An. latens formed the most basal lineage and second most basal lineage, respectively, in the Leucosphyrus group (Figure 3). Thus, the MP topology was less consistent with the traditional classification of the Leucosphyrus group in that neither the Dirus complex nor the Leucosphyrus complex and the Leucosphyrus subgroup were regarded as monophyletic taxa.

\section{Discussion}

\section{Recognition of a possible cryptic species}

For the last 30 years, Vietnamese medical entomologists [21-23] have noted that mosquitoes belonging to the Leucosphyrus group collected from northern Vietnam exhib- 


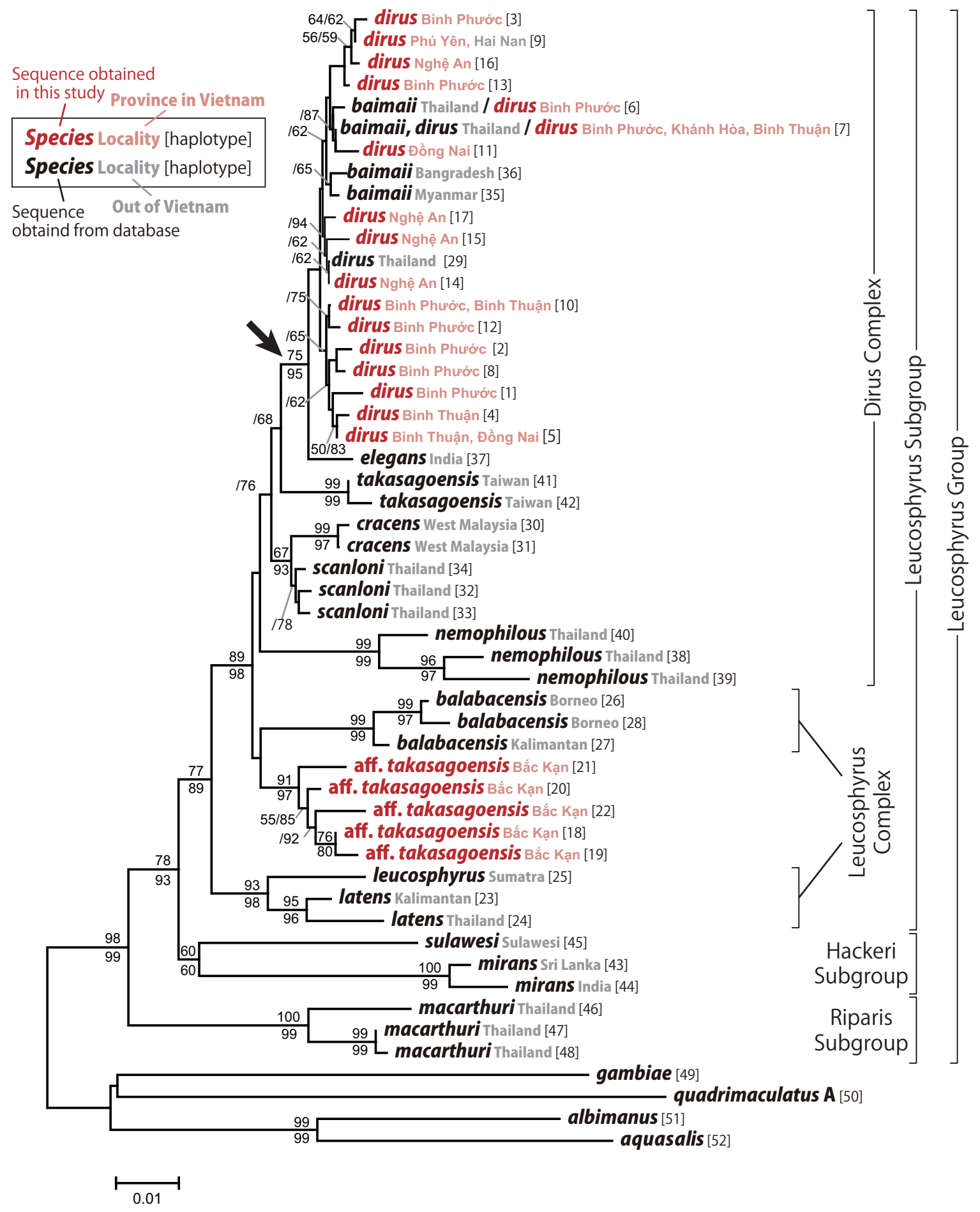

Figure 2 Neighbor-joining tree with traditional classification. The evolutionary distances were computed using the Jukes-Cantor method based on concatenated sequences of partial COI (221 bp) and ND6 (349 bp) mitochondrial genes. All positions containing missing data were eliminated only in pairwise sequence comparisons (Pairwise deletion option). The bootstrap test and the interior branch test were performed with 2,000 replicates, respectively, and each value equal to or above $50 \%$ is shown above (bootstrap value) and below (interior branch test support) the branches. 


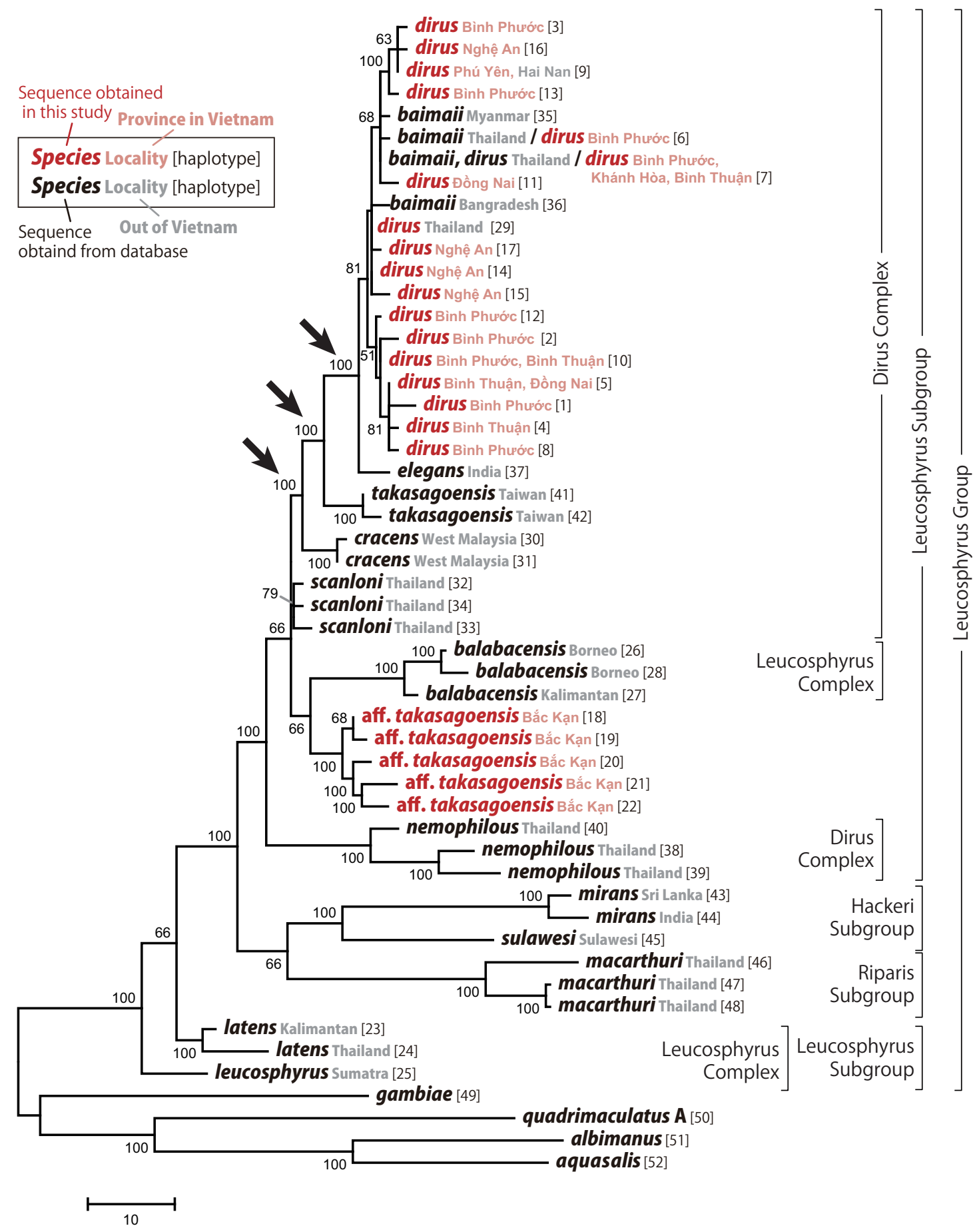

Figure 3 Maximum parsimony 50\%-majority-rule consensus tree with traditional classification. The consensus tree was generated from the 3517 most parsimonious trees based on the concatenated sequences of partial COI (221 bp) and ND6 (349 bp) mitochondrial genes. Branches corresponding to partitions reproduced in less than $50 \%$ trees are condensed. The percentages of parsimonious trees in which the associated taxa clustered together are shown next to the branches. Branch lengths were calculated using the average pathway method and are expressed in units of the number of changes over the whole sequence (scale bar). Anopheles gambiae, An. quadrimaculatus A, An. albimanus, and An. aquasalis are assigned as out-group taxa. 
ited wing-spot patterns that are different from those of An. dirus from southern Vietnam, but similar to those of An. takasagoensis, which has actually only been found in Taiwan. However, the spot pattern variations partially overlap between An. dirus and An. takasagoensis so that it is generally difficult to determine these species solely on the basis of morphological characteristics [9]. This seems to be the very reason why foreign scientists recognized that among the Leucosphyrus group members, $A n$. dirus is the only species that is distributed in Vietnam.

In the present study, all the haplotypes of the mosquitoes from southern and central Vietnam clustered into the (An. dirus + An. baimaii) clade. Although partial sequences of $C O I$ and ND6 in mitochondrial DNA do not provide a clear distinction between An. dirus and An. baimaii [25], [29], it is reasonable to regard the samples from southern and central Vietnam as An. dirus after taking into consideration the well known distributions of $A n$. dirus and An. baimaii [25], [29] (Molecular identification in Table 1). However, molecular phylogenetic analyses in this study could not resolve population structure of $A n$. dirus in Vietnam. This is also the limitation of the molecular markers and beyond the scope of the study so that we refrain from discussing the population structures of $A n$. dirus in Vietnam at present.

The haplotypes of samples collected from Bắc Kạn Province in northern Vietnam were clearly separated from those of both An. dirus and An. takasagoensis in both the NJ and MP trees. In the NJ tree, the Bắc Kạn samples formed a distinct clade with $91 \%$ bootstrap and 97\% interior branch test support, whereas the (An. dirus + An. baimaii) clade formed another clade with An. elegans with $75 \%$ bootstrap support and $95 \%$ interior branch test support (indicated by an arrow in Figure 2). In the MP tree, the Bắc Kạn samples again formed a distinct clade with $100 \%$ consensus, whereas the (An. dirus $+A n$. baimaii) clade formed another clade with An. elegans with $100 \%$ consensus, and this clade subsequently formed other clades with An. takasagoensis and An. cracens with $100 \%$ consensuses, respectively (indicated by arrows in Figure 3). These results suggest that the Bắc Kạn samples are distinctly separated from $A n$. dirus by at least three valid species--An. elegans, An. takasagoensis, and An. cracens--and from An. takasagoensis by at least one valid species--An. cracens. The clade consisting of Bắc Kạn samples formed another clade with An. balabacensis; however, the reliability of the branch was not high, with 66\% consensus in the MP tree and less than 50\% bootstrap and interior branch test support in the NJ tree. The overall morphological characteristics of the Bắc Kạn samples, however, were closest to or even indistinguishable from those of An. takasagoensis and An. dirus but were distinguishable from those of An. balabacensis and the other Leucosphyrus group members (Figure 1).
Moreover, the distribution of An. balabacensis is known to be restricted to the area from the Philippines up to Indonesia.

These results suggest that the mosquito samples obtained from Bắc Kạn Province belong to the Leucosphyrus group but not to An. dirus, An. takasagoensis, An. balabacensis, or any other species in the Leucosphyrus group; thus, these samples seem to represent a newly recognized cryptic species in the Leucosphyrus group. We tentatively designate the possible cryptic species as Anopheles aff. takasagoensis for convenience, until a valid name is assigned.

\section{Phylogenetic relationship among the Leucosphyrus group members}

In the NJ tree, the possible cryptic species formed a clade together with An. balabacensis and members of the Dirus complex with $89 \%$ bootstrap and $98 \%$ interior branch test support, whereas the other Leucosphyrus complex members, namely, An. leucosphyrus and An. latens, formed another clade beside the former clade. This topology seems to be consistent with the indications by Sallum et al. [25]. They stated that morphological distinction between the Leucosphyrus and the Dirus complexes is problematic because some characters used to define the limits of each species complex are polymorphic. Generally, members of the Leucosphyrus complex can be easily distinguished from those of the Dirus complex by the presence of an accessory sector pale (ASP) wing spot on veins $C$, subcosta, and $R$ and the absence of pale scales at the base of hind tarsomere 4 [25]. However, An. balabacensis is polymorphic for these characters and thus can overlap with members of both the Dirus complex and Leucosphyrus complex [25].

In the MP tree, the (An. aff. takasagoensis + An. balabacensis + members of the Dirus complex) clade was also supported by $100 \%$ consensus, whereas the topology within the clade was consistent with that observed in the case of the NJ tree only for the (( $A n$. dirus, An. baimaii) An. elegans) An. takasagoensis) relationship. Moreover, An. leucosphyrus and An. latens were separated from the other members of the Leucosphyrus subgroup and formed the most basal lineage and second most basal lineage in the Leucosphyrus group, respectively. This might be partly because of the long-branch attraction, to which the MP method is more sensitive than the NJ method with a corrected distance model is. It is not possible to correct for multiple nucleotide substitutions at the same site in the MP method; this leads to systematic underestimation of the genetic distances. Hence, distant species will either be clustered together or drawn toward the root of the tree [30], [31]. However, this basal positioning of An. leucosphyrus and An. latens was also reproduced by phylogenetic reconstruction using the maximum likelihood and Bayesian methods in our preliminary analyses 
(data not shown). This indicates the limitations of the present dataset: the length of the sequence data is limited, and it includes 13 of 20 species in the Leucosphyrus group whereas including all the species from various locality is desirable.

Although the information is limited, we would like to propose following three hypotheses to be tested in the future studies. First, An. nemophilous should be removed from the Dirus complex. The remaining members of the Dirus complex are then characterized by morphological characteristics in that pale scales on anterior veins of wing, especially those on presector pale and sector pale spots of the costa, are white and contrasting with other yellowish to golden pale spots on remaining posterior veins [9] (but An. aff. takasagoensis has the same characteristics). Second, members of the newly hypothesized Dirus complex (An. dirus, An. cracens, An. scanloni, An. baimaii, An. elegans, and An. takasagoensis) and An. nemophilous, An. balabacensis and An. aff. takasagoensis further form a distinct taxonomical group that is equivalent to a subgroup. Third, An. leucosphyrus and An. latens belong to the most basal or even an outer group of the remaining members of the Leucosphyrus subgroup analyzed in this study.

\section{Morphology of the cryptic species}

The cryptic species exhibited morphological characteristics distinguishable from those of typical An. dirus in southern Vietnam: the presector dark spot on vein $\mathrm{R}$ that does not extend or barely extends basally beyond the presector pale spot on the costa, a pale fringe spot present between veins $1 \mathrm{~A}$ and $\mathrm{Cu}_{2}$, and an accessory sector pale spot on the subcosta on at least one wing.

We must note, however, that these characteristics are still included within the intraspecific morphological variation of An. dirus[9]. Samples from Nghệ An Province in central Vietnam exhibited the same morphological characteristics of the cryptic species, but their haplotypes were placed within the monophyletic clade consisting of An. dirus haplotypes. The samples collected from Ninh Bình Province in northern Vietnam and analyzed by Manguin et al. ([24], mentioned in Background), with morphological characteristics of both An. dirus and $A n$. takasagoensis, might have been individuals of this $A n$. dirus type. The geographical proximity of Ninh Bình and Nghẹ An provinces supports this speculation. It is known that wing-spot patterns of Anopheles mosquitoes can vary according to temperature and day-length [32]. The wing-spot patterns of An. dirus might also vary along with the longitude in Vietnam.

\section{Distribution of the species}

According to the collection records based on identification using wing-spot patterns, populations of the hypo- thetical cryptic species have been shrinking after the 1970s, presumably because of deforestation in northern Vietnam (NDM, personal observation). Samples of the putative cryptic species have been sporadically collected from central and northern Vietnam. In 1970, 14 larvae were collected from a rice field surrounded by a forest in Cúc Phuong National Park in Ninh Bình Province. The resultant nine larval and five pupal exuviae and nine female-adult specimens are deposited in NIMPE, even though the each exuviae is mounted on a slide grass and the each adult specimens is encapsulated in a glass tube and is not available for genetic analyses. In 1973, less than 10 adult females were collected by human bate from Hòa An District, Cao Báng Province, which is located along the northern border with China (NDM, personal communication). In 2001, the putative cryptic species was collected from Yên Thành Commune, Quang Bình District, Hà Giang Province, which is also located along the northern border with China (Le Xuan Hoi, personal communication). Also in 2001, the putative cryptic species is collected from Chiêng Yên commune, Môc Châu District, Son La Province, which is located along northern-western border with Laos. Other samples are also collected from Trương Son commune, Luong Son District and Phúc San commune, Mai Châu District in Hòa Bình Province in northern Vietnam. Taking the information above and the results of molecular analyses in Manguin et $a l$. [24] and the present study into consideration, $A n$. aff. takasagoensis seems to replace $A n$. dirus in the north of Ninh Bình Province (about $20^{\circ} \mathrm{N}$ ). However, it is unclear whether An. dirus and An. aff. takasagoensis are distributed sympatrically. Further confirmation using molecular markers is necessary.

Bắc Kạn Province, from where An. aff. takasagoensis samples were collected in this study, is located near the border of Vietnam and China. Anopheles baimaii occurs in Yunnan Province in China along the borders of Laos and Myanmar [33]. Walton et al. [34] showed that the ITS2 sequence of the Chinese "species D" (An. dirus species $\mathrm{D}$ or An. baimaii) of $\mathrm{Xu}$ and $\mathrm{Qu}$ [35] is distinct from that of specimens collected in Thailand and suggested that the Chinese "species D" may represent an unrecognized species of the Dirus complex. We, however, failed to obtain consistent ITS2 sequences from our samples over the course of the present study. Confirmation of the genetic identities of An. aff. takasagoensis and the putative An. baimaii from the areas along the Vietnam, China, Laos, and Myanmar borders is also necessary.

\section{Biology of the possible cryptic species}

The larval habitat of An. aff. takasagoensis was similar to that of $A n$. dirus in southern Vietnam as described in Methods. However, the population density of $A n$. aff. takasagoensis was extremely low so that we obtained only 
11 larvae during the field collection for one week with seven staff members, even though we targeted on only this species. Moreover, the existence of the samples were localized; we found the samples from only one commune among four communes investigated.

In allozyme analyses of lactate dehydrogenase (LDH), glutamate-oxaloacetate transaminase (GOT), glucose phosphomutase (GPM), and glucose-6-phosphate dehydrogenase (G6PDH), other specimens of putative $A n$. aff. takasagoensis also exhibited a different banding pattern from that of An. dirus in southern Vietnam (NTHN et al., unpublished data). In 2006, a 10 staff-member team of NIMPE were able to collect only five female adults in a buffalo hat over a one-month field-collection period in the same study area in Bắc Kạn (Le Xuan Hoi et al., personal communication). In 2007, Manh et al. collected two female adults of the putative cryptic species at the same buffalo hat. We succeeded to obtain partial COI (but not ND6) sequences of these two samples, and the haplotypes clustered with those of other An. aff. takasagoensis specimens analyzed in the present study (data not shown). These female adults seem to have been attracted by the buffalo. NDM, one of the co-authors of this study, failed in his attempt to feed an adult female with his blood in order to obtain progeny. This implies that the hypothetical cryptic species tends to be zoophilic, although in general, An. dirus is a highly anthropophilic species. This information reinforces our hypothesis that the mosquito population from northern Vietnam belongs to a cryptic species. Further investigations of such as karyotypes and polytene chromosome banding patterns are necessary to confirm whether An. aff. takasagoensis is a valid species.

\section{Conclusions}

Morphological examination and molecular phylogenetic analyses of the members of the Leucosphyrus group in Vietnam suggested the existence of a cryptic species that is morphologically similar to, but genetically distant from both An. dirus and An. takasagoensis. We tentatively designated the species as Anopheles aff. takasagoensis. However, it was difficult to identify the species solely on the basis of morphological characteristics. Further studies on such as polytene chromosome banding patterns and karyotypes are necessary to confirm whether $A n$. aff. takasagoensis is a valid species. Further studies on the (1) geographic distribution, which is potentially spreading along the Vietnam, China, Laos, and Myanmar borders; (2) morphological and ecological characteristics; and (3) vectorial capacity of this newly identified possible cryptic species of An. dirus, which is one of the most important malaria vectors in mainland Southeast Asia, are necessary for efficient malaria vector control in this region.

\section{Additional material}

Additional file 1 Alignment of partial sequences (221 bp) of the mitochondrial $\mathrm{COI}$ gene used in this study. Every sequence is presented with the haplotype number and specific name that represents the sequence (c.f. Tables 1, 2 and 3). The consensus sequence indicates the most common bases for each site. Disagreement from the consensus sequence at each site is highlighted. Missing data are represented by an "N." Sequences of Anopheles aff. takasagoensis are surrounded by a frame.

Additional file 2 Alignment of partial sequences (349 bp) of the mitochondrial DNA ND6 gene used in this study. Every sequence is presented with the haplotype number and specific name that represents the sequence (c.f. Tables 1, 2 and 3). The consensus sequence indicates the most common bases for each site. Disagreement from the consensus sequence at each site is highlighted. Missing data are represented by an "N." Sequences of Anopheles aff. takasagoensis are surrounded by a frame.

Additional file 3 Alignment of concatenated partial sequences (570 bp in total) of the mitochondrial DNA COI and ND6 genes in FASTA format.

\section{Competing interests}

The authors declare that they have no competing interests.

\section{Authors' contributions}

KTT planned the study, conducted the field sampling of An. aff. takasagoensis in 2008 and molecular analyses, and drafted the manuscript. NTHN planned the study, conducted molecular analyses, and critically reviewed the manuscript. NTHB planned the study and critically reviewed the manuscript. TS directed the field sampling of An. aff. takasagoensis in 2008 through his expertise in the collection of larvae of the Dirus complex. He also conducted a preliminary investigation of the sampling field using GIS and critically reviewed the manuscript. MY contributed his expertise in molecular analyses and critically reviewed the manuscript. NDM planned the study; contributed his expertise in malaria vector control in Vietnam; collected, identified, and selected the samples; and critically reviewed the manuscript. MT planned the study, contributed his expertise in malaria vector control in Southeast Asia, and critically reviewed the manuscript. All authors read and approved the final manuscript.

\section{Acknowledgements}

We thank members of the Bắc Kan Provincial Malaria Center, Commune Health Center, and the Department of Molecular Biology of NIMPE, and TSUZUKI Ataru for help in the field collection in Bắc Kạn Province, Vietnam; Ho Dinh TRUNG, Le Xuan HOI, Vu Dinh CHU, Vu Duc CHINH and other members of the Department of Entomology, NIMPE for providing Anopheles samples; SAWABE Kyoko and KOMAGATA Osamu for their advice on DNA extraction; and SIBATA Hiroki, and OKUDA Naoko for their help and guidance in DNA sequencing. This study was conducted as part of the Program of Founding Research Centers for Emerging and Reemerging Infectious Diseases of the Ministry of Education, Culture, Sports, Science and Technology of Japan. This study was also partly supported by the Vietnam Global Fund Malaria Control Project to NTHN; by "the Core University Program between Japan Society for the Promotion of Science and Vietnamese Academy of Science and Technology"; by the Global COE Program, Nagasaki University, Japan; and by a Grant-in-Aid for Scientific Research of Emerging and Reemerging Infectious Diseases from the Ministry of Health, Labor and Welfare of the Japanese Government (H18-Shinko-009). All the specimens of An. aff. takasagoensis are deposited in the Department of Molecular Biology, National Institute of Malariology, Parasitology and Entomology, Hanoi, Vietnam.

\section{Author Details}

${ }^{1}$ Institute of Tropical Medicine (NEKKEN), Nagasaki University, Sakamoto, Nagasaki 852-8523, Japan, 2National Institute of Malariology, Parasitology and Entomology (NIMPE), BC 10-200, Tu Liem, Hanoi, Vietnam and ${ }^{3}$ Graduate School of Biomedical Sciences, Nagasaki University

Received: 24 February 2010 Accepted: 30 April 2010 Published: 30 April 2010 


\section{References}

1. Asian Collaborative Training Network for Malaria [http:// www.actmalaria.net/home/epidemiological profile.php\#base]

2. Institute of Tropical Medicine, Antwerp, Belgium and National Institute of Malariology, Parasitology and Entomology, Hanoi, Vietnam: Background. International Colloquium Malaria control in the Mekong Region: challenges and opportunities: 2007 2007, 3: [http://www.itg.be/internet/collog2007/] abstracts.pdf]. Hanoi, Vietnam Medical Publishing House

3. Erhart A, Thang ND, Van Ky P, Tinh TT, Van Overmeir C, Speybroeck N, Obsomer V, Hung LX, Thuan LK, Coosemans M, D'alessandro U: Epidemiology of forest malaria in central Vietnam: A large scale crosssectional survey. Malaria J 2005, 4:58.

4. Thang ND, Erhart A, Speybroeck N, Hung LX, Thuan LK, Hung CT, Ky PV, Coosemans M, D'Alessandro U: Malaria in central Vietnam: Analysis of risk factors by multivariate analysis and classification tree models. Malaria J 2008, 7:28

5. Najera JA, Zaim M: Malaria vector control: Insecticides for indoor residual spraying. WHO/CDSIWHOPES/20013 2001:102.

6. Trung HD, Bortel WV, Sochantha T, Keokenchanh K, Briët OJT, Coosemans M: Behavioural heterogeneity of Anopheles species in ecologically different localities in Southeast Asia: a challenge for vector control. Trop Med Inter Health 2005, 10:251-261.

7. Harbach RE: The classification of genus Anopheles (Diptera: Culicidae): a working hypothesis of phylogenetic relationships. Bull Entomol Res 2004, 94:537-553.

8. Sallum MAM, Peyton EL, Wilkerson RC: Six new species of the Anopheles leucosphyrus group, reinterpretation of An. elegans and vector implications. Med Vet Entomol 2005, 19:158-199.

9. Sallum MAM, Peyton EL, Harrison BA, Wilkerson RC: Revision of the Leucosphyrus group of Anopheles (Cellia) (Diptera, Culicidae). Rev Brasil Entomol 2005, 49(Suppl 1):1-152.

10. Manguin S, Garros C, Dusfour I, Harbach RE, Coosemans M: Bionomics, taxonomy, and distribution of the major malaria vector taxa of Anopheles subgenus Cellia in Southeast Asia: An updated review. 2008, 8:489-503.

11. Peyton EL, Harrison BA: Anopheles (Cellia)dirus, a new species of the Leucosphyrus Group from Thailand (Diptera: Culicidae). Mosq Syst 1979, 11:40-52.

12. Peyton EL, Harrison BA: Anopheles (Cellia) takasagoensis Morishita 1946, an additional species in the Balabacensis Complex of Southeast Asia (Diptera: Culicidae). Mosq Syst 1980, 12:335-347.

13. Baimai V, Harrison BA, Somchit L: Karyotype differentiation of three anopheline taxa in the Balabacensis Complex of Southeast Asia (Diptera: Culicidae). Genetica 1981, 57:81-86.

14. Hii JLK: Laboratory studies of three member species of the Anopheles balabacensis complex (Diptera: Culicidae). In PhD Thesis University of London; 1982

15. Baimai V, Andre RG, Harrison BA, Kijchalao U, Panthusiri L: Crossing and chromosomal evidence for two additional sibling species within the taxon Anopheles dirus Peyton and Harrison (Diptera: Culicidae) in Thailand. Proc Entomol Soc Washington 1987, 89:157-166.

16. Baimai V, Harbach RE, Kijchalao U: Cytogenetic evidence for a fifth species within the taxon Anopheles dirus in Thailand. JAm Mosa Control Assoc 1988, 4:333-338.

17. Peyton EL, Ramalingam S: Anopheles (Cellia) nemophilous, a new species of the Leucosphyrus Group from peninsular Malaysia and Thailand (Diptera: Culicidae). Mosq Syst 1988, 20:273-299.

18. Sawadipanich Y, Baimai V, Harrison BA: Anopheles dirus species E chromosomal and crossing evidence for another member of the dirus complex. J Am Mosa Control Assoc 1990, 6:477-481.

19. Peyton EL: A new classification for the Leucosphyrus Group of Anopheles (Cellia). Mosa Syst 1989, 21:197-205.

20. Obsomer V, Defourny P, Coosemans M: The Anopheles dirus complex: Spatial distribution and environmental drivers. Malaria J 2007, 6:

21. Tran DH, Nguyen DM, Nguyen TV: Anopheles (Cellia) takasagoensis Morishita, 1946 in Vietnam. In The first national conference of entomology Volume 52. Hanoi, Vietnam; 1991.

22. Institute of Malariology, Parasitology and Entomology: Keys to the Anopheles in Vietnam (Adults-Pupae-Larvae) (in Vietnamese). Hanoi: Department of Entomology, Institute of Malariology, Parasitology and Entomology; 1987.
23. Bảng ịnh loại muỗi Anophelinae ở Việt nam [http://www.nimpe.vn/ downloadnimpe/bangdinhloaimuoi.pdf

24. Manguin S, Kengne P, Sonnier L, Harbach RE, Baimai V, Trung HD, Coosemans M: SCAR markers and multiplex PCR-based identification of isomorphic species in the Anopheles dirus complex in Southeast Asia. Med Vet Entomol 2002, 16:46-54.

25. Sallum MAM, Foster PG, Li C, Sithiprasasna R, Wilkerson RC: Phylogeny of the Leucosphyrus Group of Anopheles (Cellia) (Diptera: Culicidae) based on mitochondrial gene sequences. Ann Entomol Soc Am 2007, 100:27-35.

26. Belkin JN: The mosquitoes of the South Pacific (Diptera, Culicidae) Volume 1\&2. Berkeley: University of California Press; 1962.

27. Wilkerson RC, Peyton EL: Standardized nomenclature for the costal wing spots of the genus Anopheles and other spotted-winged mosquitoes (Diptera: Culicidae). J Med Entomol 1990, 27:207-224.

28. Tamura K, Dudley J, Nei M, Kumar S: MEGA4: Molecular Evolutionary Genetics Analysis (MEGA) software version 4.0. Mol Biol Evol 2007, 24:1596-1599.

29. O'Loughlin SM, Okabayashi T, Honda M, Kitazoe Y, Kishino H, Somboon P, Sochantha T, Nambanya S, Saikia PK, Dev V, Walton C: Complex population history of two Anopheles dirus mosquito species in Southeast Asia suggests the influence of Pleistocene climate change rather than human-mediated effects. J Evol Biol 2008, 21:1555-1569.

30. Vandamme A-M: Basic concepts of molecular evolution. In The phylogenetic handbook Second edition. Edited by: Lemey P, Salemi M, Vandamme A-M. Cambridge, UK: Cambridge University Press; 2009:3-29.

31. Peer Y Van de: Phylogenetic inference based on distance methods: Theory. In The phylogenetic handbook Second edition. Edited by: Lemey P, Salemi M, Vandamme A-M. Cambridge, UK: Cambridge University Press; 2009:142-160

32. Higa Y, Toma T, Miyagi I, Malanganisho WLM, Takagi M: Morphotaxonomical studies on two subspecies of Anopheles saperoi (Diptera: Culicidae) from the Ryukyu Archipelago, Japan. Med Entomol Zool 1998, 49:207-216

33. Defence Pest Management Information Analysis Center: Regional disease vector ecology profile. East Asia. 2002 [http://www.afpmb.org/coweb/ guidance targets/disease vector/EastAsiaDVEP.pdf]. Washington DC Armed Forces Pest Management Board, Forest Glen Section, Walter Read Army Medical Center

34. Walton C, Handley JM, Kuvangkadilok C, Collins FH, Harbach RE, Baimai V, Butlin RK: Identification of five species of the Anopheles dirus complex from Thailand, using allele-specific polymerase chain reaction. Med Vet Entomol 1999, 13:24-32

35. Xu JN, Qu FY: Ribosomal DNA difference between species A and D of the Anopheles dirus complex of mosquitoes from China. Med Vet Entomol 1997, 11:134-138.

doi: 10.1186/1756-3305-3-41

Cite this article as: Takano et al., Partial mitochondrial DNA sequences suggest the existence of a cryptic species within the Leucosphyrus group of the genus Anopheles (Diptera: Culicidae), forest malaria vectors, in northern Vietnam Parasites \& Vectors 2010, 3:41

\section{Submit your next manuscript to BioMed Central and take full advantage of:}

- Convenient online submission

- Thorough peer review

- No space constraints or color figure charges

- Immediate publication on acceptance

- Inclusion in PubMed, CAS, Scopus and Google Scholar

- Research which is freely available for redistribution 